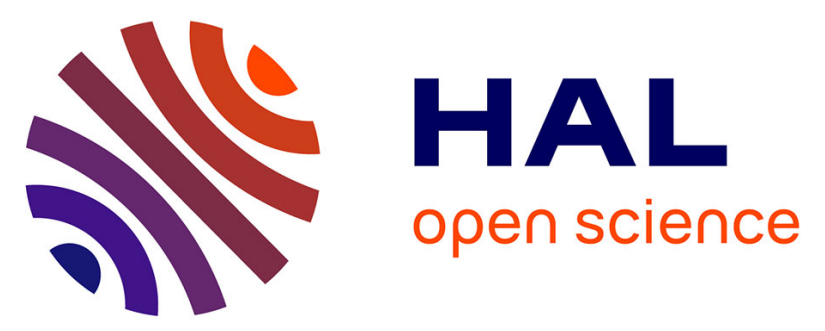

\title{
Le paradoxe des équipements sportifs. Concurrences de justifications et ségrégation socio-spatiale: Une lecture à partir du cas de Calais (France)
}

Guillaume Duchateau, Christophe Gibout, Guy Verschave

\section{- To cite this version:}

Guillaume Duchateau, Christophe Gibout, Guy Verschave. Le paradoxe des équipements sportifs. Concurrences de justifications et ségrégation socio-spatiale: Une lecture à partir du cas de Calais (France). Loisir et Société / Society and Leisure, 2016, 39 (1), pp.87-103. 10.1080/07053436.2016.1151218. hal-02383190

\section{HAL Id: hal-02383190 \\ https://hal.science/hal-02383190}

Submitted on 29 Nov 2019

HAL is a multi-disciplinary open access archive for the deposit and dissemination of scientific research documents, whether they are published or not. The documents may come from teaching and research institutions in France or abroad, or from public or private research centers.
L'archive ouverte pluridisciplinaire HAL, est destinée au dépôt et à la diffusion de documents scientifiques de niveau recherche, publiés ou non, émanant des établissements d'enseignement et de recherche français ou étrangers, des laboratoires publics ou privés. 


\section{Le paradoxe des équipements sportifs. Concurrences de justifications et ségrégation socio-spatiale : une lecture à partir du cas de Calais (France)}

\section{Guillaume Duchateau, Christophe Gibout \& Guy Verschave}

To cite this article: Guillaume Duchateau, Christophe Gibout \& Guy Verschave (2016) Le paradoxe des équipements sportifs. Concurrences de justifications et ségrégation sociospatiale ; une lecture à partir du cas de Calais (France), Loisir et Société / Society and Leisure, 39:1, 87-103, DOl: 10.1080/07053436.2016.1151218

To link to this article: http://dx.dol.org/10.1080/07053436.2016.1151218

Published online: 06 Apr 2016.

Submit your article to this journal G

LIII Article views: 2

ai

View related articles [?

View Crossmark data 巨

Full Terms \& Conditions of access and use can be found at http://www.tandfonline.com/action/journallsformation?journalCode=rles20 


\title{
Le paradoxe des équipements sportifs. Concurrences de justifications et ségrégation socio-spatiale : une lecture à partir du cas de Calais (France)
}

\author{
Guillaume Duchateau $^{\mathrm{a} *}$, Christophe Gibout ${ }^{\mathrm{b}}$ et Guy Verschave ${ }^{\mathrm{c}}$ \\ ${ }^{a}$ Université du Littoral, Côté d'Opale et Ville de Calais; ${ }^{b}$ Laboratoire TVES (EA4477), Université du \\ Littoral, Côte d'Opale; ' Université du Littoral Côte d'Opale
}

(Received 27 November 2014; accepted 1 June 2015)

\begin{abstract}
Based on the study of three sports facilities located in two marginalized neighborhoods and one middle-class district of Calais (France), this article uses, first and foremost, Boltanski and Thévenot's sociological pragmatic theory in order to analyze the reasoning behind the establishment of these facilities and the uses made of them. It shows how, and to what extent, the town councilors and other actors give meaning to their actions, and thus highlights different kinds of discrimination and social and spatial segregation.
\end{abstract}

Keywords: community facilities; sports facilities; justification; segregation; Calais

Prenant appui sur l'étude de trois équipements sportifs situés dans deux quartiers de la relégation sociale et un quartier bourgeois de la ville de Calais (France), cette étude mobilise principalement le cadre théorique pragmatiste de Boltanski et Thévenot afin de déconstruire les discours de justification de l'implantation et de l'usage de ces lieux. Elle montre comment et combien le discours des édiles et autres acteurs de l'urbain donne sens à leurs actes et, ainsi, permet de mettre au jour des formes de discrimination et d'instruire des formes de ségrégation socio-spatiale.

Mots clés : équipements collectifs; installations sportives; justifications; ségrégation; Calais

Dès les années 1930 et plus massivement depuis les années 1960 , l'équipement sportif est devenu un équipement public, collectif, produit pour une demande sociale de masse selon des logiques d'aménagement du territoire - national et local - de plus en plus rationalisées et normalisées (Bayeux, 2011). Ainsi, pour le sport comme pour d'autres services et activités des collectivités territoriales, cette logique se traduit directement dans la production de la ville (Merle, 2008). Aujourd'hui plus encore, les collectivités territoriales prennent en charge la construction et l'entretien des équipements sportifs et doivent répondre aux demandes les plus diversifiées. Certes des subventions de l'État sont accordées selon les projets, mais la part d'investissement des collectivités est devenue très importante. De plus, longtemps conçu exclusivement pour les besoins du système scolaire et du mouvement sportif, l'équipement s'ouvre depuis quelques années à des usagers - beaucoup plus diversifiés quant à leurs origines socioculturelles et à leurs finalités de pratique(s) ludosportive(s) (Ministère Jeunesse, Sport, Vie Associative, INSEE, 2005) - afin de répondre à des besoins collectifs (Bayeux, 2011).

\footnotetext{
*Corresponding author. Email: guillaume.duchateau@univ-littoral.fr
} 
Basée sur de l'analyse documentaire, des observations in situ et des entretiens compréhensifs avec des dirigeants associatifs, des élus locaux et des usagers des équipements concernés, ${ }^{1}$ cette recherche monographique s'appuie sur des éléments de la politique d'équipements sportifs de la ville de Calais dont il s'agit de déconstruire les logiques internes et externes d'implantation et de fonctionnement. Trois objets sont supports de l'étude. D'une part sur la mise à disposition de deux équipements sportifs dédiés à une politique sociale et d'autre part sur la construction d'un terrain synthétique de hockey sur gazon uniquement réservé au club local de hockey sur gazon. Les deux équipements dédiés à la politique sociale sont implantés sur les deux quartiers de la ville placés en zone urbaine sensible (ZUS), à l'Est le Beau-Marais et à l'Ouest le FortNieulay, tous deux caractérisés par une situation de pauvreté humaine définie comme « la négation des opportunités et des perspectives fondamentales sur lesquelles repose tout développement humain, à savoir vivre une vie longue, saine, constructive, et jouir d'un niveau de vie décent, ainsi que de la liberté, de la dignité, du respect de soi-même et d'autrui » (Programme des Nations unies pour le développement, 1997, pp. 15-16). Ces deux équipements ont été pensés comme une réponse publique à cette situation objective de pauvreté observée dans ces quartiers en considérant, en adéquation à la loi sur le sport du 6 juillet 2000, que « les activités physiques et sportives constitu[ai]ent un élément important de l'éducation, de la culture, de l'intégration et de la vie sociale $»$. Le terrain de hockey/gazon est implanté à Calais nord dans un quartier résidentiel et chargé d'histoire à proximité de la plage. Sa logique d'implantation est radicalement différente de celle des deux autres équipements, tant par l'usage exclusif par un sport inscrit dans une philosophie aristocratique et de distinction (Bourdieu, 1980) qui en est fait que par la structuration culturelle notabiliaire du club concerné qui a directement influé sur la décision publique de rénovation et d'affectation de l'équipement (Schéma 1).

Pour expliquer ce matériau, nous avons choisi d'avoir prioritairement recours au cadre théorique de la justification (Boltanski et Thévenot, 1991). Ce demier permet de poser la question des accords et des controverses, en se fondant sur des principes supérieurs communs - ou Cités ${ }^{3}$ - qui les organisent afin de les mettre en cohérence interme et externe pour asseoir une concorde au sein de l'être-ensemble. En reliant les exigences de l'accord aux conditions de la discorde, ce modèle théorique nous permet de mieux pointer la situation paradoxale des équipements sportifs consistants, ex ante, en une concurrence de justifications et, ex post, en la fabrique d'une ségrégation socio-spatiale, appuyée sur une double stigmatisation (Goffman, 1975) : une étiquette péjorant un quartier à l'intérieur de l'agglomération calaisienne, puis un triple processus de discréditation à l'intérieur même des quartiers de la relégation urbaine.

Confrontant matériau empirique et modèle théorique autour de cette question des enjeux de l'équipement collectif et de service public, ici sportif, nous assistons alors au télescopage entre des logiques politiques et des formes concurrentes de justification. Ce sont ce télescopage et ses conséquences dont il convient ici de démêler les entrelacements afin de comprendre des causalités propres aux politiques publiques et de « poser la question de la capacité de la ville à faire société » (Donzelot, 1999, p. 87).

\section{Présentation de la ville de Calais}

Ville littorale située sur la Côte d'Opale, Calais est frontalière avec la Grande-Bretagne; son port passager occupe d'ailleurs la première place nationale et la deuxième place européenne. La ville de Calais est la plus peuplée du Département du Pas-de-Calais avec ses 77317 habitants et constitue la ville-centre d'une agglomération du Calaisis de près de 100000 habitants. 
C'est une ville au passé industriel très présent, économiquement pauvre et marquée par un fort taux de chômage et de précarité. L'organisation urbaine de la ville est caractérisée par un morcellement de son territoire, renforcé par la présence de canaux qui le traversent, et un paysage urbain contrasté aux formes d'habitat disparates. On constate une reconstruction non maîtrisée après les bombardements de la Seconde Guerre mondiale qui l'avaient détruite à $90 \%$ ainsi qu'une urbanisation contrastée et en ilots de la Ville au fil des « trente glorieuses " qui ont eu pour effet que l'on a pour habitude de définir Calais comme étant « une Ville de villes».

\section{Contexte socio-économique}

Calais se distingue, au niveau de la structure sociale de sa population, par une surreprésentation des jeunes par rapport au reste de la région et de la France : 29,6\% des habitants sont âgés de moins de 20 ans (contre $24,8 \%$ en France et $28 \%$ en région Nord-Pas-de-Calais). Le taux d'accroissement de la population est le deuxième de la région. Le revenu fiscal moyen médian s'élève à 13318 euros en 2008 (contre 17632 sur le territoire national, INSEE ${ }^{4} 2009$ ) et $68 \%$ des foyers sont attributaires des minima sociaux. Le taux de chômage est deux fois plus élevé que la moyenne nationale $(21,7 \%$ à Calais contre $10,4 \%$ en France, INSEE décembre 2014). Le pourcentage de personnes percevant l'allocation parent isolé est quatre fois supérieur à la moyenne nationale.

L'économie a été durablement marquée par les effets de l'ouverture, dans les années 1990, du tunnel sous la Manche. Cette ouverture a généré à l'époque une croissance d'emplois à Calais; néanmoins, après une phase de stabilisation, on constate depuis les années 2000 une très forte récession économique, imputable - au moins en partie - à la structure industrielle de la ville.

Traditionnellement l'activité économique à Calais était orientée sur les activités portuaires, la dentelle, la chimie, le commerce de détail et le transport (passagers et marchandises). Depuis le début des années 2000, ces industries connaissent des changements que personne n'a su anticiper. Les chocs industriels sont conséquents et tout un pan de l'industrie tend à disparaître : 162 licenciements sur 251 salariés dans la principale entreprise chimique calaisienne (Calaire Chimie) entre 2012 et 2013 (La Voix du Nord, 07/ 01/2015), emplois dans la dentelle passant de 45000 dans l'entre-deux-guerres (dans 110 usines) à 1500 en 2009 (dans 15 entreprises) (Le Monde, 01/08/2008), baisse de $25 \%$ de l'activité transport et logistique entre 2013 et 2014 (CCI ${ }^{5}$ Côte d'Opale, 2014), etc.

Tout ceci a pour conséquence un fort taux de chômage, qui situe Calais dans les tout premiers rangs nationaux faisant de cette ville, un exemple typique des " nouveaux territoires du chômage » (Bissuel, 2013). Sa répartition est inégale selon les quartiers : plus forte dans les quartiers ZUS du Beau-Marais et du Fort-Nieulay (taux avoisinant les $40 \%$, INSEE $2007^{6}$ ). Cette situation touche plus particulièrement les jeunes; dans ces quartiers le taux de chômage des 15-24 ans est de plus de $60 \%$.

La ville de Calais est très marquée par deux types de population : une minorité de gens aisés issus de la prospérité liée à la dentelle ou aux services publics (Sous-Préfecture, collectivités locales, Chambre de Commerce et d'Industrie, enseignements public et privé secondaire et supérieur, etc.) et une majorité de gens pauvres au regard d'une acception pluridimensionnelle de la pauvreté : revenu, santé, structures sociale et familiale, éducation et illettrisme.

La ventilation des ménages selon les catégories socioprofessionnelles fait apparaître davantage d'ouvriers ( $28 \%$ ) et dans une moindre mesure d'employés $(12 \%)$ parmi les ménages calaisiens qu'au niveau de la région ( $25 \%$ ouvriers et $11 \%$ employés). En 
revanche les cadres y sont moins nombreux ( $5 \%$ à Calais contre plus de $7 \%$ dans la région).

On note également une forte proportion de familles monoparentales et notamment de jeunes femmes, souvent jeunes et vivant seules avec plusieurs enfants. La pauvreté culturelle est très forte. L'illettrisme touche un pourcentage important de la population (27\% dans les ZUS, $12 \%$ en Région Nord-Pas-de-Calais contre $7 \%$ en France, INSEE 2012, Rapport ONZUS $^{7}$ 2013). Les populations souffrant d'addiction(s) sont surreprésentées au regard de la population nationale. En particulier dans les quartiers défavorisés de 1'Est et de l'Ouest de la ville, nombre de ménages connaissent enfin des situations de chômage et d'assistanat les conduisant à des phénomènes de " désocialisation " et d'exclusion sociale (Coignet, 2013; Gibout, 2012; Vieille Marchiset et Coignet, 2011).

Le pourcentage des populations issues de l'immigration reste très faible, avec moins de $4 \%$ d'étrangers résidents (principalement d'origine algérienne, marocaine et turque). L'immigration clandestine est en revanche très importante, puisque sont notés plus de 41000 passages par an (transits vers la Grande-Bretagne) et quelque 1500 repas servis chaque jour aux migrants.

\section{Politique sportive de la ville de Calais}

Calais est dotée d'un tissu associatif sportif assez riche, elle compte 85 associations sportives subventionnées par la ville, regroupant, au total, à peu près 10000 licenciés. La ville compte cinq clubs de haut niveau (deux volley-ball, deux basket-ball, boxe anglaise) ainsi que quelques clubs de niveau intermédiaire (CFA en football ce qui correspond à la quatrième division, hockey sur gazon en seconde division). La quantification des pratiquants auto-organisés (Chantelat, Fodimbi, et Camy, 1996) ou « sauvages » (Mauny et Gibout, 2008) est plus aléatoire, mais la commune dispose de plusieurs sites dédiés à ces pratiques - à la fréquentation tendanciellement avérée par plusieurs études ainsi que des occupations ludosportives de lieux originellement non dédiés à ces modes de faire : footing à la plage ou autour de la citadelle édifiée selon les plans de Vauban, " football des trottoirs" (Sansot, 2009) ou skateboard sur différentes places publiques, etc.

Historiquement, à l'instar d'un grand nombre de collectivités territoriales (Bourg et Nys, 2012), la ville a investi une politique sportive par le financement d'équipements sportifs et d'associations, par l'organisation de manifestations sportives, par le prêt de matériels afín de soutenir la vie associative, ou encore par la mise à disposition d'équipements sportifs au bénéfice des établissements scolaires du premier et du second degré. De sorte qu'à l'exception de quelques évènements sportifs, la ville a développé insidieusement une « logique de guichet » (Honta, 2008; Voléry, 2008) où les associations locales étaient invitées/incitées à solliciter sur un mode de l'interconnaissance, de la connivence ou encore de l'automaticité tacite de la reconduction des subventions et autres aides de la part des collectivités territoriales. Nous entendons par cette terminologie le fait que les clubs et autres associations sportives subventionnées par la municipalité s'adressent à la commune uniquement pour solliciter des créneaux aux seins des équipements ou encore pour l'obtention de subventions de fonctionnement ou destinées à l'organisation d'une manifestation sportive souvent d'intérêt local. La collectivité a toujours encouragé cette « logique de guichet ». En effet, elle n'a jamais sollicité les clubs autrement ou quant à d'autres objectifs, d'autres stratégies ou finalités - i.e. éducatives, sociales, de solidarité, d'intégration, de développement territorial, de développement économique, etc. - de sa 
politique territoriale. Ainsi que le développe Vincent Dubois (2010), cette « politique au guichet, politique du guichet » a conduit à la mise en cuvre d'un pouvoir tendanciellement discrétionnaire et à une discrimination des classes populaires par la bureaucratie; ce mode de gouvernement aboutissant à une forme de régulation des pauvres par l'État social.

Depuis 1999-2000, les territoires sont en profonde évolution sous l'effet conjugué des lois Voynet et Chevènement ${ }^{8}$ mais aussi sous l'effet du mouvement progressif et continu de décentralisation. On assiste ainsi à une véritable " recomposition territoriale " qui se manifeste notamment par le renforcement des politiques sportives territoriales (Bourg et Nys, 2012; Honta, 2008). Les collectivités territoriales et leurs groupements ont de plus en plus de latitude pour concevoir et mettre en cuvre les politiques de développement local.

À cet égard, le cas calaisien est assez allégorique de cette bascule dans une logique plus entrepreneuriale et participative. À l'instar de ce qui s'observe également ailleurs, apparaissent, à partir des années 2000 de nouvelles initiatives territoriales qui peu à peu entendent transformer l'espace du polity - les institutions de gouvernance et les choix collectifs liant des parties - par la mise en place de procédures locales d'implication accrue de la société civile (Nonjon, 2000) et celui du policy - les " problèmes » dans différents secteurs de l'intervention publique - par l'élaboration de nouveaux agencements entre les politiques sectorielles et les politiques locales préalablement développées (Balme, Faure, et Mabileau, 1999). Dans le cadre de la politique sportive, cela se traduit par des redéfinitions de l'action publique locale, des sollicitations accrues du tissu associatif pour des finalités extra-sportives et des initiatives à l'échelle inter-institutionnelle (Honta, 2004).

En 2008, le mouvement prend une dimension véritablement accrue pour ce qui concerne le cas calaisien. La municipalité communiste en place depuis 37 ans est battue et on voit arriver une jeune maire de droite qui veut davantage axer son action dans une logique libérale et basée sur la performance, l'innovation et l'entrepreneuriat. Avec ce changement de municipalité, la politique sportive est en partie réorientée à la fois autour de la promotion du sport d'élite comme vecteur de notoriété territoriale (soutien financier accru aux clubs de haut niveau) mais aussi sur la base ambitieuse d'un vaste projet éducatif intitulé « Éducation par le Sport ». Le budget du sport de la ville est alors passé de $6,5 \%$ à 7,5\% par rapport au budget total entre 2008 et 2014 (cf. tableau 1).

Ainsi, dans le cadre de la politique de la ville et grâce au soutien financier des contrats urbain de cohésion sociale, le recrutement d'une équipe de 10 éducateurs sportifs a permis d'assoir cette politique sur les trois quartiers sensibles de la ville. En 2013, ce sont donc 1 200 adolescents sans licence sportive et issus des secteurs les plus défavorisés de la ville qui fréquentent les activités proposées dans ce dispositif initié exclusivement au sein de ZUS. Des partenariats forts avec l'Agence pour l'Éducation par le Sport qui - avec le Comité interministériel à la ville - avait initié entre 2007 et 2011 une recherche-action sur le territoire des ZUS de la commune (Coignet, 2013; Vieille Marchiset et Coignet, 2011),

Tableau 1. Subventionnement des clubs de sports de la ville de Calais entre 2005 et 2014.

\begin{tabular}{lcrr}
\hline Subventions & 2005 & 2009 & \multicolumn{1}{c}{2014} \\
\hline Clubs haut niveau & 1155762 & 1385447 & 1442200 \\
Clubs sportifs de masse & 322218 & 385814 & 386815 \\
\hline
\end{tabular}


la Protection judiciaire de la Jeunesse, l'Éducation nationale à travers la plateforme de décrochage scolaire ont permis de développer des actions pertinentes au vu des problématiques locales. Ce dispositif expérimental d'éducation par le sport bénéficie d'une certaine reconnaissance au niveau national dans la mesure où, au motif de l'identification de son fort potentiel dans les quartiers prioritaires, il est publicisé dans les réseaux institutionnels et professionnels (i.e. journée Association des Maires de France en 2008, Colloques au Sénat en 2013 et 2014) et où il bénéficie d'un financement récurrent de la part de l'État dans le cadre de la politique de la ville.

\section{Justification d'une politique sportive sur le fondement de la clause générale de compétence}

L'histoire du sport territorial est étroitement liée à la construction des premiers équipements, notamment pour les scolaires puis pour les clubs sportifs. C'est là une étape décisive dans la définition première d'une politique municipale des sports puis dans la structuration des services municipaux des sports et parfois, sur un mode complémentaire, des offices municipaux des sports (Bayeux, 2011).

Les compétences des collectivités territoriales en matière sportive ne sont pas clairement définies (Ministère Ville, Jeunesse et Sports, 2014), cependant, les collectivités territoriales assument aujourd'hui la responsabilité de plus de $80 \%$ des équipements sportifs français.

Les communes sont considérées comme un des principaux financeurs du sport en France puisqu'elles y consacrent chaque année près de 7 milliards d'euros, ce qui en fait le premier financeur public du sport en France. Paradoxalement, aucun texte - pas plus les lois de décentralisation depuis les années 1980 que la loi du 16 juillet 1984 sur la promotion et l'organisation du sport en France ou le code du sport (version consolidée au 7 décembre 2014, ministère de la Ville, de la Jeunesse et des Sports) - n'oblige les communes à intervenir dans le domaine sportif. Ce principe connaît une exception : la prise en charge des équipements sportifs nécessaires à la pratique de l'éducation physique et sportive (EPS) ${ }^{9}$. Dès lors, l'intervention de la municipalité dans le domaine sportif se fait sur le fondement de la clause générale de compétence.

En application de l'article L.212-29 du Code général des collectivités territoriales, « le conseil municipal règle, par ses délibérations, les affaires de la commune ». Sur ce principe, il appartient donc à ce conseil municipal de définir sa politique et les moyens qu'il entend y consacrer. Cette grande liberté laissée à l'initiative des élus locaux explique très certainement la disparité des politiques sportives sur le territoire français.

La prise en charge significative du sport par les communes date des années 1930, mais elle a surtout subi une accélération au cours des IVe et Ve plans (1961-1965, 1970-1975) ${ }^{10}$ avec les lois de programme d'équipements sportifs et socio-éducatifs. Cette politique volontariste a permis au pays de se doter d'un patrimoine sportif. Aujourd'hui, avec une moyenne de $6 \%$ à $7 \%$ de leur budget consacré au sport (CNFPT, 2002), les communes développent des politiques qui vont au-delà de la gestion de ce patrimoine et qui s'articulent autour de quatre grands domaines d'intervention (Ministère Ville, Jeunesse et Sports, 2014).

- Les équipements : il s'agit de la construction, de la gestion et de la mise à disposition des équipements sportifs.

- Le soutien : essentiellement orienté vers les associations sportives, il prend la forme de subventions ou de mise à disposition de personnels.

- L'animation : grâce à du personnel spécialisé dans l'encadrement des activités physiques et sportives, les communes développent, sur les temps scolaire, périscolaire et 
extrascolaire, des politiques éducatives sportives en partenariat avec l'école et les associations sportives.

- La promotion, les manifestations : les communes participent activement à l'organisation des manifestations sportives, le plus souvent avec les associations implantées localement.

Si nous choisissons d'articuler cette rapide présentation avec la théorie des justifications et le modèle des Cités cher à Boltanski et Thévenot (1991) qui permettent de porter l'attention sur les dynamiques d'engagement et les régimes d'action publique, nous constatons, dans le cas de Calais, une adéquation des politiques municipales des sports avec, simultanément, la " Cité civique » et la " Cité industrielle ». En effet, les valeurs d'accord qui justifient l'action publique sportive sont d'abord la Démocratie, la Solidarité, l'Équité et la Liberté puis, secondairement, l'Efficacité et la Science. En effet, ce sont d'abord l'élection des municipalités et la délégation qu'elles ont reçue du Peuple, ensuite l'expertise, la rationalité et le professionnalisme des administrations locales qui sont supposés être les garants d'une lecture juste de la situation, d'une mise en œuvre efficace et plus encore d'une légitimité de l'action publique sportive.

Basé jusqu'alors sur le principe de cette clause générale de compétence et sur ce principe de double justification de l'initiative publique, le parc d'équipements sportifs traditionnels calaisiens se voit donc quelque peu bousculé par l'érection des trois équipements qui constituent le matériau de cette analyse.

Ainsi, en 2011 et 2013, deux nouvelles salles de sports sortent de terre, appuyées sur des logiques d'implantation inédites à l'échelon local. Composés d'un plateau multisports et d'une salle d'expression culturelle destinée à assoir l'action phare de sa politique sportive à caractère social et dédiés aux adolescents des quartiers sensibles, ces équipements ne sont absolument pas destinés à recevoir des compétitions sportives et les associations sportives traditionnelles n'en sont pas utilisatrices. En effet, la municipalité réserve les créneaux au sein de ces salles uniquement aux associations de quartiers ainsi qu'aux éducateurs sportifs municipaux ceuvrant dans le cadre de cette nouvelle politique d'éducation par le sport. La justification qui fonde cette double implantation adjoint alors au modèle précédent - en particulier la logique d'équité et de solidarité essentielle à la "Cité civique »-une référence à la « Cité par projets » basée sur l'extension des réseaux sociaux, la prolifération des liens, la flexibilité et l'autonomie (Boltanski et Chiapello, 1999). Le bien commun partageable est alors triplement justifié dans le discours des édiles et son niveau de référence au sein du débat varie selon les autres acteurs en présence et leur degré d'accord avec ses justifications. Ainsi, face aux clubs pérennes du quartier qui revendiquent des créneaux d'occupation de ces nouvelles installations, la municipalité répond par une délocalisation de l'activité de ces associations sportives hors du quartier qui ne remet pas en cause sa propre logique d'action publique.

En parallèle de la construction de ces équipements socio-sportifs, un terrain de hockey sur gazon répondant aux normes internationales a été inauguré. Cette installation performante est exclusivement réservée à l'unique club local de la discipline. Ce petit club évoluant en National $2^{11}$ compte 108 licenciés et occupe cette installation en moyenne une douzaine d'heures par semaine soit trois soirées, une partie du mercredi après-midi et un week-end sur deux. Cet aménagement s'inscrit en continuité d'une politique nationale comme locale où, depuis le début de la $V^{e}$ République, le paysage urbain était marqué par le développement des équipements sportifs normés pour la compétition (Augustin, 1995). Ainsi, la majorité des salles et terrains de sports répondent aujourd'hui aux cahiers des charges des règlements fédéraux afin de permettre la pratique compétitive pour les clubs locaux. Mais, plus encore dans le cas précis du terrain de hockey sur gazon calaisien, cet 
équipement semble jouer d'un triple registre de justifications (Boltanski et Thévenot, 1991) qui trouve à se déployer dans les discours des acteurs en présence, organise et référence les formes de l'accord (ou du désaccord) possible entre eux quant à l'existence même ou à l'usage de ce terrain. D'une part, il reprend à son compte le principe de justification et de rationalisation du discours inhérent au modèle de la « Cité industrielle » basé sur l'efficacité, la performance et la productivité - ici celles inhérentes au haut niveau sportif -, mais il leur rattache une justification par la "Cité domestique ", à savoir un registre de raisonnement reposant sur les valeurs classiques de la famille, de la tradition, de la discrétion et de la loyauté. C'est d'abord l'attention pour la " gagne », les « battants » et l'inscription dans la logique de rivalité et de compétition qui épuisent le discours du nouvel adjoint aux sports lors de l'inauguration. C'est ensuite, plus en sourdine, mais diffus dans le discours des dirigeants du dit-club, la référence à la tradition locale, aux familles qui fondent depuis le dix-neuvième siècle la bourgeoisie locale, à la distinction et à la noblesse de la pratique (Pinçon-Charlot et Pinçon, 1996, 1997). " Du coup [pour ce terrain comme pour les deux autres équipements] les situations sont des nouds lors desquels les enjeux prennent forme \# (Boissonade, 2009, p. 44).

Ainsi, si nous considérons les trois équipements concemés par notre étude, nous observons que la logique la plus traditionnelle se retrouve à Calais Nord pour une pratique sportive fédérale et compétitive qui se déroule sur le terrain de hockey et dans le club-house adjacent. En opposition à cela, nous rencontrons une logique plus récente et plus innovante qui s'exprime à travers une pratique sportive libre et/ou éducative, mais non fédérée dans les salles multi-activités Gauguin-Matisse et Nelson Mandela, équipements à peine plus anciens situés dans les deux principaux quartiers défavorisés de la ville. Nous sommes conséquemment confrontés à une problématique de contradictions non antagoniques, les paradoxes inhérents à l'équipement sportif dédié à une forme de pratique. D'une part, le dispositif socio-sportif réservé à un public dans le cadre d'une politique sociale et d'autre part l'équipement dédié à une forme de pratique sportive (en l'occurrence le hockey sur gazon). Mais, au-delà des écarts et/ou des oppositions de justifications qui posent la question d'une mise en tensions interne à la politique sportive de la ville de Calais telle qu'elle s'exprime dans les discours des élus et leurs échanges avec les autres acteurs locaux au fil de sa mise en cuvre, c'est aussi l'expression de formes ségrégatives à l'ceuvre sur l'ensemble des trois équipements qui est à interroger. En suivant la logique de Norbert Elias (Elias et Scotson, 1965), on peut poser l'hypothèse que la politique publique à l'origine de ces équipements met en place une politique de discrimination consciente et assumée avec le risque de stigmatisation de ces populations (Gibout, 2012).

\section{Les raisons de la mise à disposition de ces équipements sportifs dédiés à cette politique sociale}

Ces trois dernières décennies ont été marquées par le développement d'équipements sportifs en tous genres (Augustin, 1995; Bourg et Nys, 2012), notamment les structures d'accès libre destinées aux pratiques sportives auto-organisées. Nous nous tournons ici vers un exemple d'équipements dédiés aux actions d'éducation et d'intégration par le sport sous deux formes : d'une part la mise en place de créneaux en libre accès et d'autre part des séances encadrées par des éducateurs sportifs recrutés à cet effet.

En premier lieu, une volonté politique très forte de la ville de construire et de valoriser une politique éducative globale, y compris par le sport, et qui est à la base de ces constructions d'équipements. Ensuite, il y a la volonté d'attribuer des créneaux dans les salles de sports aux habitants des quartiers en difficulté. Enfin, apporter une réponse politique forte aux demandes 
des « jeunes » pour obtenir des créneaux de futsal, hip-hop (cultures urbaines)... Et globalement, cela correspond à la commande et au rôle majeur de la politique de la ville tant du point de vue financier ( $50 \%$ de financement des actions) que de la mise en ouvre sur le terrain. De plus, la politique nationale de la ville (programmes de l'Agence nationale pour la rénovation urbaine et dispositif Dotation de développement urbain) accompagne l'engagement financier de la ville dans la construction d'équipements sur les quartiers ciblés. Les dernières études du Centre national de la fonction publique territoriale (CNFPT, 2002) ou encore de l'Association nationale des élus en charge du sport (ANDES, 2005) mettent en évidence que les finalités définies comme prioritaires pour les politiques sportives territoriales sont les finalités éducatives ( $90 \%$ des communes) et d'insertion ( $70 \%$ des communes). Ces finalités éducatives et intégratives sont le soubassement des missions de service public remplies par les services des sports, mais aussi celles qui sont le plus porteuses de légitimité politique pour les élus locaux. Nous constatons donc un réinvestissement de la «justification civique » qui se trouve tempéré par un discours de responsabilisation et d'autonomisation propre à la « Cité par projets " (Boltanski et Chiapello, 1999) et qui confirme l'intrusion du laïus fondé sur l'entrepreneuriat, le coaching, la médiation et la flexibilité, lequel n'est pas sans poser des questions - craintes, protestations et interrogations - pour les associations durablement implantées dans ces " quartiers de la relégation sociale " qui ne constituent plus de véritables « sas d'adaptation à la société » (Marchal et Stébé, 2010, p. 44).

\section{Pourquoi le tissu associatif regimbe}

Ce tissu associatif dans ces quartiers répertoriés comme sensibles se compose de 19 associations sportives et représente 2085 licenciés. Ces clubs revendiquent des créneaux horaires au sein de ces équipements sportifs situés sur leur secteur de recrutement et d'implantation historique de leur club respectif. Les sièges de ces clubs se trouvent sur ces territoires périphériques au sein desquels des liens forts - d'amitié, de connivence et/ou de solidarité - ont été tissés avec les riverains (Coignet, 2013). Ces associations ne disposants pas systématiquement de créneaux proches dans les salles existantes se voient dans l'obligation de s'expatrier dans d'autres quartiers plus éloignés des quartiers en ZUS pour pratiquer leur(s) sport(s), qu'il s'agisse d'entrainements ou de compétitions. « Il faut le dire, on voulait la salle Gauguin-Matisse et la maire nous a donné Calypso ${ }^{12}$ [...] On a été déporté de l'autre côté du canal!!! » (Une dirigeante d'un club sportif du quartier du BeauMarais). Par ailleurs des associations extérieures déjà fortement financées dans d'autres cadres (en particulier celles s'inscrivant dans une logique de haut niveau) et disposant de personnels qualifiés interviennent dans le cadre de cette action d'éducation par le sport au sein de ces nouveaux équipements, ce qui ne manque pas de créer des tensions avec les populations locales et associations anciennement affiliées au(x) quartier(s). Ainsi, les clubs de haut niveau en basket-ball, volley-ball et football envoient-ils leurs éducateurs diplômés en intervention dans ses nouvelles salles, contre rémunération qui n'est alors pas directement réinvestie dans la vie économique du quartier. Les clubs des quartiers se plaignent en outre auprès des élus, par courrier ou interpellation orale, pour dénoncer une tentative de recrutement de jeunes sportifs ou une confiscation de l'identité locale. «Ils [les éducateurs] sont pas d'ici [le quartier Fort-Nieulay]. Ils occupent nos terrains de foot et ils nous piquent nos p'tits z'éfants ${ }^{13}$ " (un bénévole sportif d'un club de football du Fort-Nieulay).

Nous rencontrons ici un conflit de légitimité entre ces associations sportives locales qui revendiquent une forme renouvelée de « droit du sol » et les intervenants extérieurs éducateurs municipaux et associations non indigènes sollicitées - qui eux revendiquent l'inscription dans une politique municipale volontariste confortée par la réélection en 2014 
de l'équipe municipale en place, une forme de «légitimité du vote ». En nous référent aux travaux de Boltanski et Thévenot ordonnant la théorie des justifications (1991), nous retrouvons ici, avec un agencement renouvelé, la "Cité domestique " des clubs des quartiers (appartenance à une forme de famille, de clan ou de communauté infra-locale) et la « Cité de l'opinion » des jeunes de ces quartiers (logique de réputation et de prestige, de visibilité et de célébrité), une combinatoire entre les «Cité civique " (légitimité de l'élection), "Cité marchande » (logique de concurrence et de " gagne ») et "Cité industrielle " (principe d'efficacité et de performativité) pour les acteurs en charge des projets initiés par la municipalité en collaboration avec les services communaux et d'autres associations que celles des quartiers. Il s'agit alors de saisir les différents registres de justification en cours et, pour chacun des acteurs en présence, de déterminer son propre régime d'action, susceptible d'être pertinent moralement et en pratique, face aux autres registres convoqués in situ (Boissonade, 2009).

\section{Stigmatisation d'un public}

Même si cette politique sportive a porté ses fruits puisque le nombre d'adolescents mobilisés a considérablement augmenté entre 2010 et 2014, passant de 280 à plus de 1 200 jeunes, le tissu sportif traditionnel s'est senti lésé par ce dispositif. En effet, les associations sportives traditionnelles - fédérales et à visée compétitive - de ces secteurs géographiques n'étaient pas en mesure de s'adapter et de pouvoir recevoir ce public nouveau constitué de jeunes issus des quartiers plutôt défavorisés et éloignés de la pratique sportive de club. Ce jeune public rejetait la compétition sous sa forme institutionnelle et lui préférait des modalités plus ludiques et intermittentes (Gasparini et Vieille Marchiset, 2008). Conséquemment, ces clubs anciens ne pouvaient pas prétendre à disposer de ces équipements implantés sur leur territoire de recrutement. Ce dispositif éducatif largement relayé par les médias locaux et nationaux a quelque peu mis à l'écart le tissu associatif sportif le premier implanté dans ces quartiers prioritaires. De ce fait, ces associations sportives locales ne disposent pas de créneaux au sein de ces équipements de proximité situés sur leur secteur de recrutement et cela engendre une certaine frustration. " Le club était là avant les tours et on a toujours travaillé avec ces jeunes du quartier... On leur donne le goûter, on leur apprend les règles [...] Et maintenant on n'existe plus. La mairie, elle oublie notre travail » (un dirigeant sportif du quartier du Beau-Marais n'y résidant pas). Ces associations sportives traditionnellement présentes éprouvent un sentiment d'exclusion et de relégation, comme si était niée ou rejetée l'ancienneté de leur action au coeur des quartiers et avec des populations qu'elles ont peu à peu apprivoisées (Coignet, 2013).

De plus, les adolescents fréquentant ces installations dédiées à cette forme autoorganisée de pratiques sont montrés du doigt comme étant les seuls utilisateurs autorisés à jouir de ces équipements, suspectés de les privatiser à leur usage exclusif voire même d'en tirer un avantage de prestige ou de réputation dans le quartier. Ces adolescents, régulièrement qualifiés selon les époques de public difficile, de rebelles, de sauvageons... se retrouvent stigmatisés par le tissu associatif sportif. Ce sont des individus, et non, par extension irrégulière, les quartiers et les équipements sportifs, qui sont stigmatisés dans une relation sociale déterminée. Cette stigmatisation " tribale " (Goffman, 1975) des jeunes des quartiers par les associations locales introduit alors non pas une ségrégation du quartier de la relégation urbaine par son extérieur - ségrégation existante par ailleurs -, mais une stigmatisation à l'intérieur même du quartier entre les inclus dans la vie associative et les exclus de cette dernière. 
Par ailleurs, ces équipements sportifs sont des lieux qui valorisent majoritairement les cultures masculines et invisibilisent les jeunes filles (Deville, 2007) dans l'espace public. On retrouve uniquement le public féminin sur des créneaux qui lui sont réservés et encadrés de façon spécifique. Par ailleurs les travaux de Noria Boukhobza (2000) montrent qu'il est extrêmement difficile pour une fille dans ces quartiers populaires de pratiquer une activité sportive, elles sont idéologiquement assignées à d'autres tâches. Alors que $51 \%$ des filles pratiquent le sport en France, seulement $32 \%$ le font en zone d'éducation prioritaire (Gasparini et Vieille Marchiset, 2008). Ces lieux de pratiques dans ces deux quartiers réintroduisent la division du genre au sein de l'espace sportif. Le club traditionnel demeure l'espace sportif régulé où les filles peuvent trouver leur place et la pratique auto-organisée reste l'espace libre d'accès largement dominé par les garçons. Une division genrée de l'espace qui n'est qu'une des matérialités possibles de pratiques symboliques qui rappellent que la ville est « un miroir concret des normes de genres et elle est aussi, à travers les choix politiques, l'un des outils de la régulation sociale $»$ (Denèfle, 2004, p. 540).

À travers les actions proposées, ces équipements relativement « innovants " par rapport à la question sociale (Coignet, 2013) entrent insidieusement dans une triple logique discriminante produite par l'activité sportive : une distinction stigmatisante à l'égard du tissu associatif ancien des quartiers, une discrimination à l'égard des extérieurs au quartier par les insiders, une ségrégation à l'égard des femmes.

\section{Distinction sociale, logique de production d'équipement sportif et croisement de deux Iogíques sociétales}

Dans le même temps, la collectivité a construit, au sein de la citadelle sise au nord de la ville, un terrain de hockey sur gazon de dernière génération aux normes internationales. C'est le club local qui jouit de manière exclusive de cette installation. En parallèle, la municipalité a mis à disposition à proximité de ce terrain un club-house que l'association a magnifiquement aménagé à grand frais et qui permet tout à la fois de favoriser la sociabilité entre gens du même monde et d'entretenir la mémoire - et la légende - de l'institution via des photographies anciennes et des manifestations commémoratives (voir Photo 1 et Photo 2). Ce nouvel

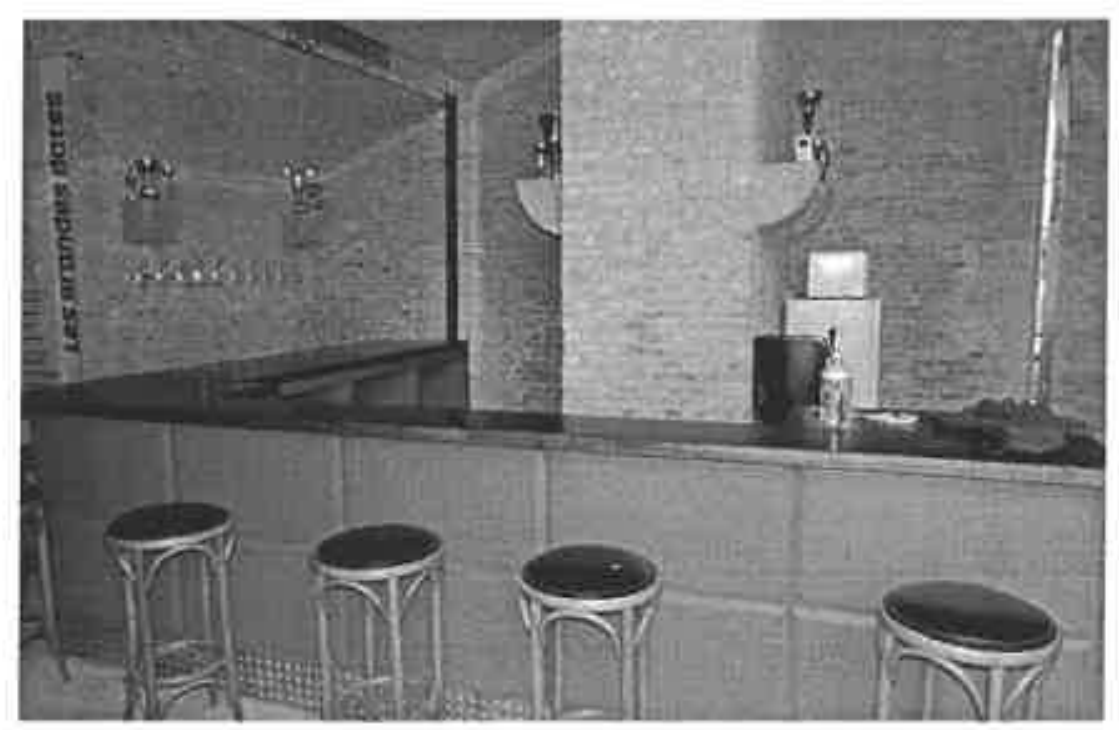

Photo 1. Vue du club-house. Photo : G.Duchateau/TVES. 


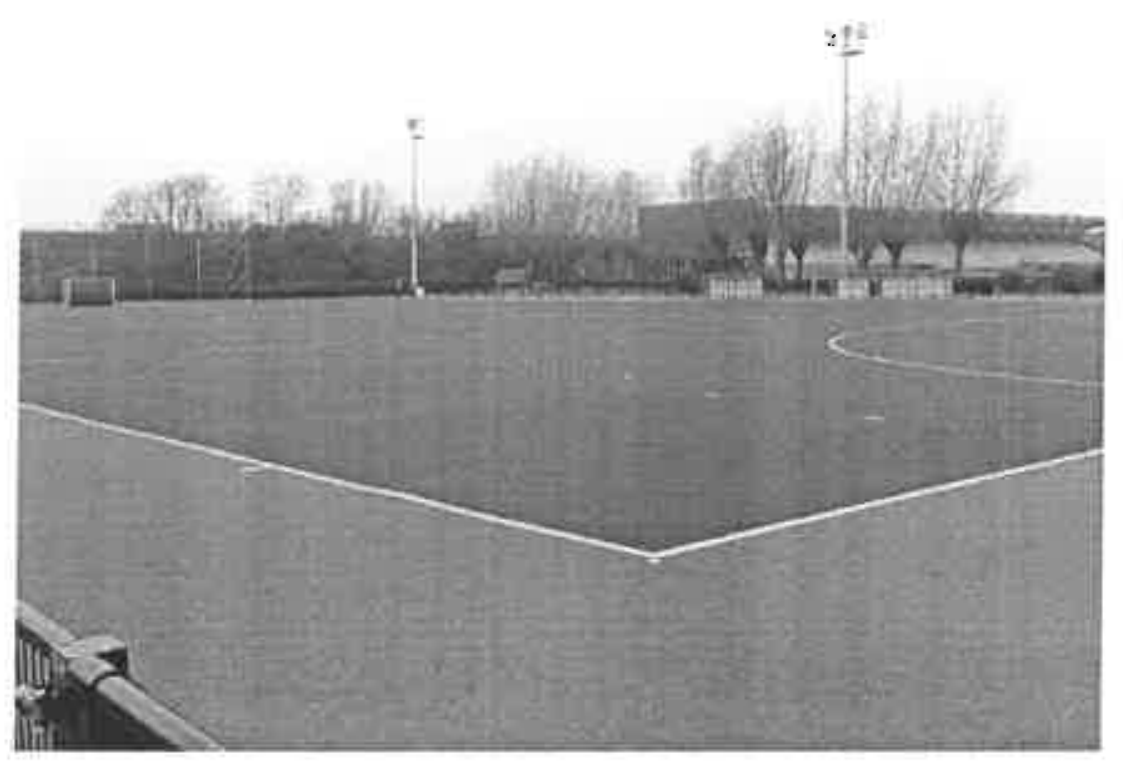

Photo 2. Vue du terrain de hockey. Photo : G.Duchateau/TVES.

équipement très couteux est donc attribué exclusivement aux licenciés du club de hockey sur gazon et à leur famille qui y cultivent leurs réseaux de notables et s'y retrouvent parfois pour fêter des événements communautaires, traiter des affaires et/ou échanger dans un cadre discret, cossu et confortable. Les dirigeants ainsi que les adhérents de cette association sont issus de la bourgeoisie calaisienne et les modes de recrutement de ce club sont proches de la cooptation et du parrainage. Le tout souscrivant ainsi aux principes de l'entre-soi bourgeois (Pinçon-Charlot et Pinçon, 1997).

Ainsi, l'influence des dirigeants de ce club a eu raison des politiques locaux et après plusieurs années de négociations et de transactions (Rémy, 2005) avec les représentants de la municipalité qu'ils côtoyaient dans d'autres cercles culturels, politiques et/ou philanthropiques, la décision de construire cet équipement fut prise au détriment des anciens utilisateurs du terrain multisports pourtant bien plus nombreux. Dans le rapport inégal de forces en présence entre les différents groupes sociaux, les groupes sportifs et la collectivité locale, le compromis s'est institué largement au profit d'un collectif (le club de hockey/gazon) dont les intérêts ont largement croisé ceux de la municipalité avec laquelle existaient des connivences de valeurs et de l'interconnaissance (Pinçon-Charlot et Pinçon, 1996). Bien qu'anciennement utilisateurs de l'équipement, les autres clubs sportifs, en situation défavorisée dans le processus de concertation et de décision, n'ont pas pu porter leur parole jusqu'au terme du mouvement d'élaboration de la décision politique et ont, conséquemment, été exclus du nouvel espace sportif.

Cet accord [s'est] construit dans une séquence temporelle d'ajustements successifs. Il y [fallait] de l'échange, de la négociation pour tenter de rapprocher les points de vue, et de l'imposition pour arbitrer quand le rapprochement s'avère impossible et qu'il faut trancher. C'est exactement la définition de la transaction sociale. (Blanc, 2000, p. 690)

Alors qu'ils mobilisaient une grammaire d'argumentation appuyée sur la " Cité civique » et le sens de l'intérêt général pourtant défendue par la municipalité, mais 


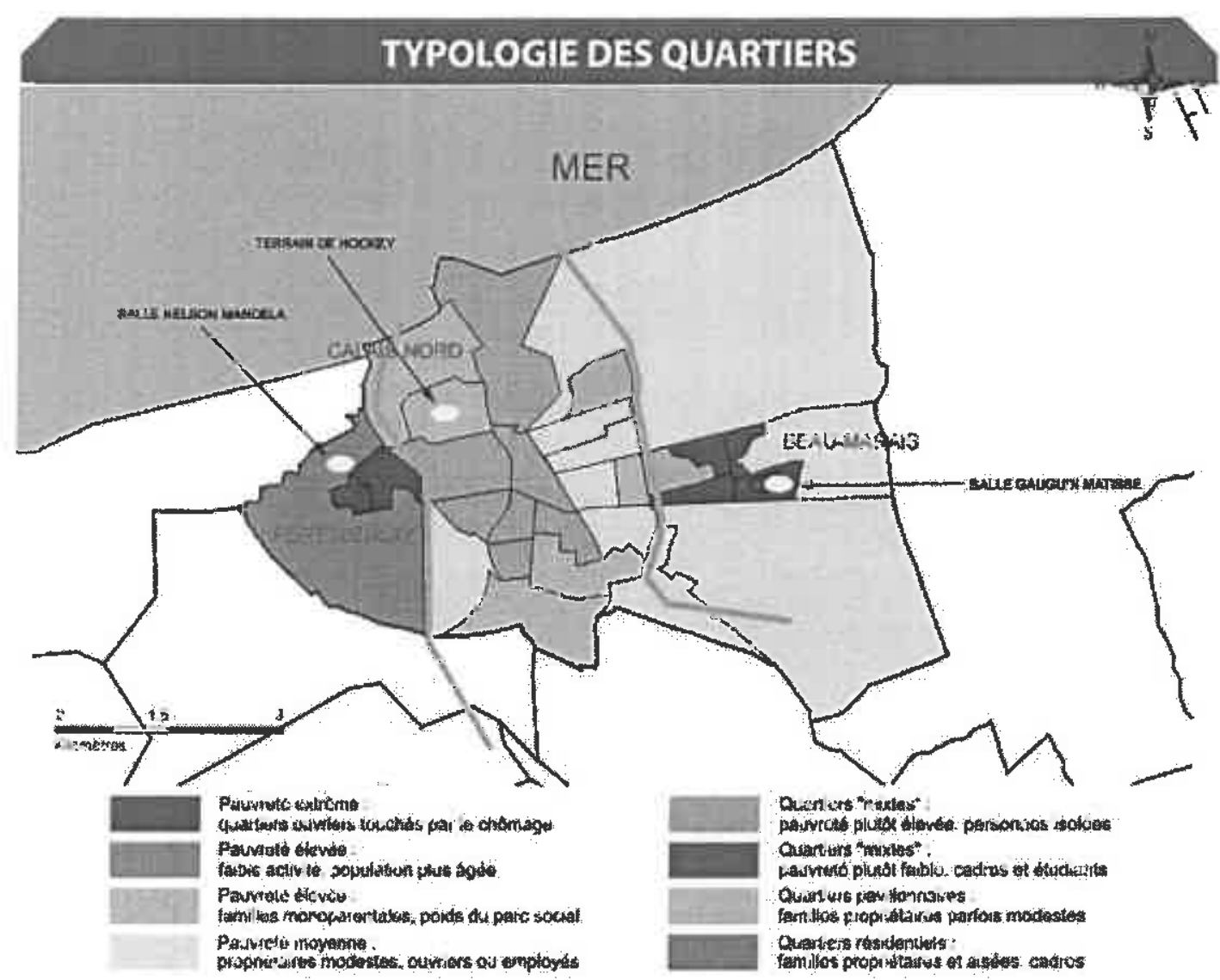

Schéma 1. Cartographie des trois équipements.

ailleurs et dans leurs échanges avec d'autres acteurs de la collectivité, le club de football et les autres associations qui utilisaient auparavant l'ancien équipement multisports en synthétique et accédaient également aux salles annexes se sont donc retrouvés relégués à l'extérieur de la citadelle. Nous observons donc que, aux motifs d'un « registre de justifications » (Boltanski et Thévenot, 1991) fondé concomitamment sur la "Cité domestique " et sur la "Cité marchande », un équipement sportif dédié construit une relégation de certains clubs et de certains groupes sociaux à l'intérieur de l'espace urbain. Cet exemple témoigne ainsi, de façon plus globale, que " les débats relatifs à la démocratie locale prennent corps au sein de sociétés dans lesquelles le rapport entre la société civile et le politique se délite progressivement " (Jouve, 2005, p. 317).

\section{Conclusion}

L'évolution de fond des politiques sportives territoriales témoignent d'une mutation profonde dans la façon de penser et de gérer le territoire aujourd'hui. Elles ont des incidences sérieuses sur les politiques d'attribution et d'utilisation des équipements sportifs. Nous retrouvons ici deux logiques sociétales qui se croisent au sein d'une même politique sportive. D'une part, l'équipement sportif attribué à un type de public pour une forme de pratique, libre, éducative et non compétitive et, d'autre part, l'attribution d'un équipement à un club sportif pour une pratique sportive de distinction sociale. 
Dans ces deux situations, le fait de réserver un équipement pour une forme de pratique ou pour un public ne fait qu'accentuer l'agacement et l'incompréhension des autres acteurs individuels et/ou collectifs en droit - du moins en espérance - de jouir également de ces installations.

Ces situations analysées témoignent de tensions et de conflits - plus ou moins ouverts, plus ou moins figés dans le temps - qui s'articulent autour des « façons dont les personnes s'engagent dans l'action, leurs justifications et le sens qu'elles donnent à leurs actes » (Boltanski et Chiapello, 1999, p. 36). Ces régimes d'action et de justification permettent de donner une cohérence interne à l'action et/ou au discours produits par les parties en présence. Mais, cette concurrence de justifications, reposant sur des grammaires de valeurs ontologiquement différentes, oblige à l'élaboration de compromis ou de transactions qui, dans le présent de l'engagement et sans abdiquer sur les valeurs propres à chaque groupe, s'apparentent davantage à de la « coopération conflictuelle » (Simmel, 2007, pp. 45-59).

Ce dont témoigne cet exemple calaisien réside dans deux possibles montées en généralité qui interpellent illico la question de la gouvernance et de la fabrique d'un système urbain, avec des éléments qui conservent une certaine autonomie, tout en étant soumis à des interdépendances croissantes.

D'une part, les politiques publiques territoriales - ici du sport - rappellent directement la nécessité de la dimension spatiale du politique ainsi que l'impératif d' " appréhender la dimension spatiale des inégalités » (Veschambre, 2012, p. 263). En effet, les enjeux liés aux services de proximité sont évidemment ceux de l'accessibilité et de l'équité - sociales et/ou spatiales -, mais, plus largement ils sont ceux de la solidarité et de la violence, de la stigmatisation et du lien, des identités sociétales et/ou communautaires ainsi que des normes et valeurs qui les sous-tendent, c'est-à-dire, pour le dire en un mot, ceux du vivre-ensemble.

D'autre part, les politiques publiques locales - ici sportives - sont prises dans des écheveaux de pressions et de conflits - latents ou manifestes -, de contradictions et de résistances qui obligent souvent les municipalités à des arbitrages qui, au fil du temps, prennent force dans des registres de justifications et des répertoires de normes et/ou de valeurs qui sont concurrentiels et clivés, à savoir de l'ordre de l'irréductible tension et du " deux dans un même lieu " (Certeau, 1984, p. 286). Plus que jamais donc, face à une demande sociétale éclatée et fluctuante, les édiles et autres dirigeants sont contraints à transiger dans l'ici et le maintenant et à faire fi d'une cohérence au long cours de leur action publique. Se pose alors, comme à d'autres échelles, la question, ancienne et toujours renouvelée, de la "[g]ouvernabilité et [des] transformations structurelles des démocraties » (Gaxie, 1996).

\section{Disclosure statement}

No potential conflict of interest was reported by the authors.

\section{Notes}

1. Le travail d'enquête a démarré en 2010 pour se terminer en 2014. Il a également bénéficié de l'implication institutionnelle - comme directeur du département Jeunesse et Sports - d'un des auteurs dans la collectivité locale depuis 2008 , ainsi que de précédentes enquêtes effectuées, pour le SG-CIV (secrétariat général du Comité interministériel des Villes) et 1'APELS (Agence pour l'éducation par le sport), entre 2007 et 2011 concernant « Les clubs sportifs, comme lieux 
d'intégration et d'éducation, dans les zones urbaines sensibles ", pour lesquelles le Calaisis avait été support d'étude privilégié.

2. Instituées par la loi du 14 novembre 1996, les ZUS sont, jusqu'en 2015, des périmètres d'application de la politique de la ville et du développement social urbain. Cette action, conduite conjointement par l'État et les collectivités territoriales, vise à identifier des quartiers prioritaires pour l'action publique afin de lutter contre les phénomènes d'exclusion, d'améliorer l'habitat dégradé et l'équipement de ces quartiers dans une optique de régénération urbaine et de mixité sociale. Sur les 750 ZUS identifiées en France en 1996, trois concernaient le territoire de la ville de Calais dont les deux quartiers (Beau-Marais et Fort-Nieulay) où sont implantés les équipements ici étudiés.

3. Dans leur ouvrage de 1991, Luc Boltanski et Laurent Thévenot (1991) proposent six cités (cité inspirée, citée domestique, cité de l'opinion, cité civique, cité marchande, cité industrielle) qui sont, chacune, fondées sur des valeurs propres, valorisent ou dévalorisent certains items et impliquent des formes différentes d'accords. Par la suite, Claudette Lafaye et Laurent Thévenot (1993) ont ajouté une septième cité dite " écologique » et Luc Boltanski et Ève Chiapello (1999) une huitième cité dite " par projet ».

4. L'NSEE est l'Institut national de la statistique et des études économiques. Il s'agit de l'organisme public français en charge des études statistiques sur le territoire national.

5. La CCI est la Chambre de Commerce et d'Industrie. Il s'agit, en France, de l'organisme consulaire organisant et réfléchissant à l'activité économique sur un territoire donné.

6. cf. http://www.insee.fr/fr/regions/nord-pas-de-calais/default.asp?page=themes/ouvrages $/ \mathrm{dev}$ durable/DVA0_07.htm.

7. L'ONZUS est l'Observatoire national des zones urbaines sensibles. Il s'agit d'un organisme d'État en charge de l'évaluation des politiques publiques dans les quartiers prioritaires.

8. La loi dite "Voynet " d'orientation sur l'aménagement et le développement durable du territoire, datant du 25 juin 1999, crée deux nouvelles entités territoriales - le pays et l'agglomération - qui doivent être porteurs d'un projet définissant les orientations de choix de développement économique et d'aménagement urbain, en particulier dans le cadre d'une contractualisation avec l'État et/ou la Région portant sur la requalification urbaine et le développement solidaire. La loi dite "Chevènement » de simplification et de renforcement de la coopération intercommunale, promulguée le 12 juillet 1999 , donne incitation au regroupement intercommunal afin que les nouveaux territoires soient pertinents et cohérents quant à la réalisation de nouveaux projets en commun.

9. L'EPS est, en France, la discipline d'enseignement qui a pour support les activités physiques, sportives et artistiques. Le corps et la motricité sont l'objet de cette discipline.

10. De 1946 à 2006, la République française a mis en place un Commissariat général au Plan en charge de définir la planification économique du pays via des plans quinquennaux définissant les grandes orientations stratégiques et les objectifs de la politique nationale à moyen et long terme.

11. Il s'agit là du quatrième niveau national de pratique pour cette activité. Le professionnalisme sportif y est totalement absent.

12. Calypso est le nom d'un complexe sportif situé à environ 2 kilomètres de la salle espérée, de l'autre côté d'un canal qui coupe le quartier du reste de la ville.

13. Les « p'tits z'éfants » signifie, en patois local, les « petits enfants d'ici ».

\section{Références}

ANDES. (2005). Les politiques sportives des villes. Balma : ANDES.

Augustin, J.-P. (1995). Sport, géographie et aménagement. Paris : Nathan.

Balme, R., Faure, A., et Mabileau, A. (dirs.). (1999). Les nouvelles politiques locales. Dynamiques de l'action publique. Paris : Presses de Sciences Po.

Bayeux, P. (2011). Le sport et les collectivités territoriales. $\left(4^{\mathrm{e} e} \mathrm{ed}\right)$. Paris : Presses universitaires de France.

Bissuel, B. (2013). Les nouveaux territoires du chômage. Le Monde. Retrieved December 26, 2013. consulté le 10 mars 2015 . from www.lemonde.fr/politique/article/2013/12/26/les-nouveaux-ter ritoires-du-chomage_4340160_823448.html 
Blanc, M. (2000). Les multiples facettes de la citoyenneté : clarification du concept et enjeu de la citoyenneté pour l'Europe. Dans Collectif, Invention et Réinvention de la Citoyenneté (pp. 683696). Pau : Éditions Joëlle Sampy.

Boissonade, J. (2009). Les apports de la sociologie pragmatique à la transaction sociale. Le concept de régimes d'action dans l'analyse du discours des jeunes de banlieue. Pensée plurielle, 20(1), 37-50. doi:10.3917/pp.020.0037

Boltanski, L., et Chiapello, E. (1999). Le nouvel esprit du capitalisme. Paris : Gallimard.

Boltanski, L., et Thévenot, L. (1991). De la justification. Les économies de la grandeur. Paris : Gallimard.

Boukhobza, N. (2000). Mémoires de filles, histoires de quartiers. Un regard ethnologique. Les cahiers du CEDREF [En ligne]. 8-9 |2000, mis en ligne le 28 août 2009, Consulté le. Retrieved septembre 08, 2014 from hitp://cedref.revues.org/203

Bourdieu, P. (1980). Comment peut-on être sportif? Dans, Questions de sociologie (pp. 173-195). Paris: Minuit.

Bourg, J.-F., et Nys, J.-F. (2012). Clubs sportifs et collectivités territoriales : Enjeux, modèles, partenariats, stratégies. Voiron : Presses universitaires du sport.

Certeau (De), M. (1984). L'invention du quotidien. Volume I : Arts de faire. Paris : Gallimard.

Chantelat, P., Fodimbi, M., et Camy, J. (1996). Sports de la Cité. Anthropologie de la jeunesse sportive. Paris : L'Harmattan.

CNFPT (2002). Les politiques sportives des villes de plus de 3000 habitants. Antenne MidiPyrénées.

Coignet, B. (2013). Sport et innovation sociale. Des associations sportives en mouvement dans les quartiers populaires. Paris : L'Harmattan.

Denèfle, S. (Coord.). (2004). Femmes et villes. Tours : Presses universitaires François Rabelais.

Deville, J. (2007). Jeunes filles « invisibles » dans les quartiers populaires. Espaces et Sociétés, 128 129(1), 39-53. doi:10.3917/esp.128.0039

Donzelot, J. (1999). La nouvelle question urbaine. Esprit, 11, 87-114.

Dubois, V. (2010). Politiques au guichet, politique du guichet. Dans D. O. Borraz, et V. Guiraudonet (dirs.), Politiques publiques, 2. Changer la société (pp, 265-286). Paris : Presses de Sciences Po.

Elias, N., et Scotson, J. L. (1965). The established and the outsiders. London: Sage Publications.

Gasparini, W., et Vieille Marchiset, G. (2008). Le sport dans les quartiers. Pratiques sociales et politiques publiques. Paris : Presses universitaires de France.

Gaxie, D. (1996). Gouvernabilité et transformations structurelles des démocraties. Dans Collectif, La gouvernabilité (pp. 249-273). Paris : Presses universitaires de France-CURAPP.

Gibout, C. (2012). Justice sociale vs justice spatiale, ou le paradoxe de l'accessibilité aux services de proximité. Dans D. R. Cortéséro (dir.), La banlieue change! Inégalités, justice sociale et action publique dans les quartiers populaires (pp. 46-62). Lormont : Le Bord de l'eau.

Goffman, E. (1975). Stigmate. Les usages sociaux des handicaps. $1^{\text {te }}$ éd. US 1963. Paris : Minuit.

Honta, M. (2004). Sport et action publique locale : Des initiatives entre concurrence et complémentarité inter-institutionnelles. Le cas de la ville de Mérignac (Gironde). Staps, 63(1), 107-122. doi:10.3917/sta.063.0107

Honta, M. (2008). Sport et intercommunalité : Quels enjeux pour la définition des politiques départementales et l'articulation de l'action publique sportive locale? Staps, 80(2), 115-136. doi: $10.3917 / \mathrm{sta} .080 .0115$

Jouve, B. (2005). avril). La démocratie en métropoles : Gouvernance, participation et citoyenneté. Revue française de science politique, 55(2), 317-337. doi:10.3917/rfsp.552.0317

Lafaye, C., et Thévenot, L. (1993). Une justification écologique? Conflits dans l'aménagement de la nature. Revue française de sociologie, 34, 495-524. doi:10.2307/3321928

Marchal, H., et Stébé, J.-M. (2010). La ville au risque du ghetto. Paris : Lavoisier.

Mauny, C., et Gibout, C. (2008). Le football " sauvage » : D'une autre pratique à une pratique autrement. Science \& Motricité, 63(1), 55-61.

Merle, S. (2008). Politique d'équipements sportifs et évolution post-industrielle en région stéphanoise. Métropoles [En ligne]. 4|2008, mis en ligne le 12 décembre 2008, consulté le 12 novembre 2014. Retrieved from http://metropoles.revues.org/3342

Ministère de la jeunesse, des sports et de la vie associative, Ministère de la culture, INSEE. (2005). La pratique des activités physiques et sportives en France. Paris : Éditions de 1'Insep. 
Ministère de la ville, de la jeunesse et des sports. (2014). Code du Sport, Consulté le 08 décembre 2014 à l'adresse suivante. Retrieved from http://www.legifrance.gouv.fr/affichCode.do? cidTexte=LEGITEXT000006071318

Nonjon, M. (2000). Participation des habitants et règles du jeu municipal. Cahiers lillois d'économie et de sociologie, 35-36, 205-221.

Pinçon-Charlot, M., et Pinçon, M. (1996). Grandes Fortunes. Dynasties familiales et formes de richesse en France. Paris : Payot.

Pinçon-Charlot, M., et Pinçon, M. (1997). Voyage en grande bourgeoisie. Paris : Presses universitaires de France.

Programme des Nations unies pour le développement. (1997). Rapport mondial sur le développement humain. Paris : Economica.

Rémy, J. (2005). Négociations et transaction sociale. Négociations, 3(1), 81-95. doi:10.3917/ neg.003.0081

Sansot, P. (2009). Le football des trottoirs. Dans, Les gens de peu (rééd., pp. 141-154). Paris : Presses universitaires de France.

Simmel, G. (2007). Les grandes villes et la vie de l'esprit (rééd ed.). Paris : L'Herne.

Veschambre, V. (2012). Appréhender la dimension spatiale des inégalités : L'accès au "conservatoire de l'espace". Dans D. B. Bret, P. Gervais-Lambony, C. Hancock, F. Landyet (dirs.), Justice et injustices spatiales (pp. 263-279). Nanterre : Presses universitaires de Paris-Ouest.

Vieille Marchiset, G., et Coignet, B. (dirs.). (2011). Les clubs sportifs dans les Zones urbaines sensibles. Des lieux d'intégration et d'éducation? Rapport final de la recherche-action 2007-2011 pour le Comité Interministériel à la Ville (CIV) et l'Agence pour l'Éducation par Le Sport (APELS). Paris : CIV/APELS.

Voléry, I. (2008). Sociogenèse d'un mode de gouvernance territoriale. Le cas d'un contrat éducatif local. Revue française de science politique, 58(5), 743-771. doi:10.3917/rfsp.585.0743 